\title{
Energy Efficient Segmentation-Link Strategies for Transparent IP over WDM Core Networks
}

\author{
Mohd Nazri Mohd Warip ${ }^{1}$, Ivan Andonovic ${ }^{2}$, Ivan Glesk ${ }^{2}$, Phaklen Ehkan ${ }^{1}$, Fairul Afzal Ahmad Fuad ${ }^{1}$, and \\ Mohamed Elshaikh Elobaid Said Ahmed ${ }^{1}$ \\ ${ }^{1}$ School of Computer and Communication Engineering, University Malaysia Perlis, Pauh Putra Campus, 02600 Arau, \\ Perlis, Malaysia \\ ${ }^{2}$ Deparment of Electronic and Electrical Engineering, University of Strathclyde, 204 George Street, Glasgow G1 1XW, \\ United Kingdom \\ Email: nazriwarip@unimap.edu.my; i.andonovic@strath.ac.uk; i.glesk@strath.ac.uk; phaklen@unimap.edu.my; \\ fairul@unimap.edu.my; elshaikh@unimap.edu.my
}

\begin{abstract}
Recent developments in Optical IP networks have heightened the need for reduction on power consumption via so called green photonics and concepts known as green networking. Adhering to these principles we proposed a novel energy savings approach which can be applied to optical IP networks and is based on so called "hibernation mode with segmentation link" technique. The hibernation mode technique is designed to help to reduce power consumption by sleep the network links and can help to optimise network overall energy usage by taking advantage of the hibernation algorithms we have developed. The proper implementation over the network infrastructure will save energy, operating cost to network operators, and while at the same time will also deliver reduced carbon footprint. In this paper, we show design model for developing energy efficient Segment-Link approach. In this scheme, the improvised energy saving techniques focus on selective sleeping of optical core fibre links, following which the Routing Wavelength Assignment algorithm based on GMPLS control plane are investigated. Simulation models and results for the proposed scheme are evaluated in both the fibre link only and segmentation-link schemes scenarios. The results shows that a significant amount of energy can be saved if appropriate "segmentation-link" mechanisms using optical bypass and traffic grooming is invoked.
\end{abstract}

Index Terms - Energy efficiency, IP over WDM networks, power consumption, OXC, GMPLS, segmentation-link

\section{INTRODUCTION}

One of the most significant Internets today became an integral part of everyday lives in the modern society. It constantly fuels growing opportunities for new and more advanced services designed to better respond to our always changing needs. However, there are certain drawbacks associated with the introduction of a new service or hardware would often be done without thinking too much about its impact on the environment. By our standards today, such approach would be in the strongest contrast with today's global drive for energy efficiency.

Manuscript received July 09, 2013; revised September 17, 2013.

This work was supported by the School of Computer and Communication Engineering, Universiti Malaysia Perlis (UniMAP) under Grant No. RAGS 9018-0003.

Corresponding author email: phaklen@unimap.edu.my.

doi:10.12720/jcm.9.1.48-55
Since its advent, the Internet has impetus a significant part of global economic growth. In fact, the global internet traffic increases 1.3 times every year, or quadruples every five years. Such unprecedented surge of internet traffic is attributed to newly emerging internet access technologies, such as mobile access infrastructure and various new network-oriented applications such as online video services, file sharing services, and web services. Increase in internet traffic brings a significant issue of the energy consumption problem as well as the network capacity problem [1]. Defined by its interconnections and routing policies and underpinned by a higher level optical infrastructure, it has fuelled the increased demands for the provisioning of new and more advanced services able to dynamically react to changes within the network. These newly developed services are varied and consume ever more amounts of energy, counter to the global drive for a greener environment. Consequently there is an evolving need for an optical infrastructure that complies with zero-carbon emission principles.

The continuing deployment and upgrade of optical telecommunication networks rapidly drive up power and energy consumption, in a way that makes operators worry that future energy consumption levels may pose constraints on communications growth that are more stringent than those posed by bandwidth limitations [2]. In addition, the current core networks are overdimensioned in terms of switching capacity, the number of deployed links and nodes to meet network growth and reliability constraints. The introduction of novel lowconsumption silicon technologies is on its own not sufficient for migrating the current network towards greener operation. There are opportunities therefore to explore the possibility of adapting network energy requirements to the actual traffic profiles saving network operational costs as well as reducing the carbon footprint [3].

In this paper we discuss an approach which can help to reduce power consumption in IP over optical networks and will optimise their energy efficiency by implementing the developed hibernation algorithm with 
segmentation-link schemes. Its implementation can help to safe on network operational cost as well as reduce the carbon footprint.

The rest of this paper is organized as follows; in Section II, potential energy saving and power consumption contributions in transparent Optical IP networks are introduced. Section III presents network energy model and energy saving duration process. Section IV introduces segmentation-link mechanisms adopted in hibernation mode techniques. Section V provides simulation results and Finally Section VI concludes this paper.

\section{POTENTIAL ENERGY SAVING IN TRANSPARENT CORE IP OVER WDM NETWORKS}

One of the most significant cost reduction strategies in core optical IP networks (also sometimes known as IP over Optical or IP over WDM) in terms of provisioning, operation and maintenance is savings in energy consumption. The major power consumption contributors of equipment in the network are:

- WDM chassis; receiving/transmitting equipment such as transponders modules, short-reach optical interface transponders.

- Optical Switching (OXC) Chassis, opaque/transparent optical transponders, Optical-Electrical-Optical (OEO) conversion.

- Core Router: electronic processing, traffic grooming and aggregation in the IP layer.

- Optical Amplifiers: predominately the Erbium Doped Fibre Amplifier (EDFAs).

- 3R Regenerators: signal regeneration with re-timing, re-amplification and re-shaping operations.
- Control Plane: signalling and routing algorithm modification.

\section{A. Power Consumption Contributions}

The key to designing energy efficient transparent IP over optical [4], [5] transport network are rooted in the network architecture.

The characterisation of the energy consumption in any of these architectures requires metrics that capture the main elements that consume energy;

$\mathrm{E}_{\mathrm{G}}$ : Energy consumed by Core IP Router when performing routing or grooming operations in the electronic domain

$\mathrm{E}_{\mathrm{W}}$ : Set of WDMs which consume energy

$\mathrm{E}_{\mathrm{X}}$ : Energy dissipated by OXCs

$\mathrm{E}_{\mathrm{TR}}$ : Energy consumption of each WDM transponder

$\mathrm{E}_{\mathrm{A}}$ : Energy consumption of optical amplifiers (viz. EDFA) within WDM spans placed at $70 \mathrm{~km}$ intervals.

\section{B. Transparent IP over WDM Architecture}

Transparent Optical IP is defined as a network whereby optical signals are transported from source to destination entirely in the optical domain (without OEO conversion) [5]. Therefore, optical channels can bypass intermediate nodes and because of this, signal regeneration is not required.

Each node in a transparent IP over optical network is typically equipped with a Micro-Electro-Mechanical System (MEMS)-based [4] OXC/reconfigurable optical add-drop multiplexer (ROADM) [4], [5] directly linked to a core router through WDM transponders (Fig. 1).

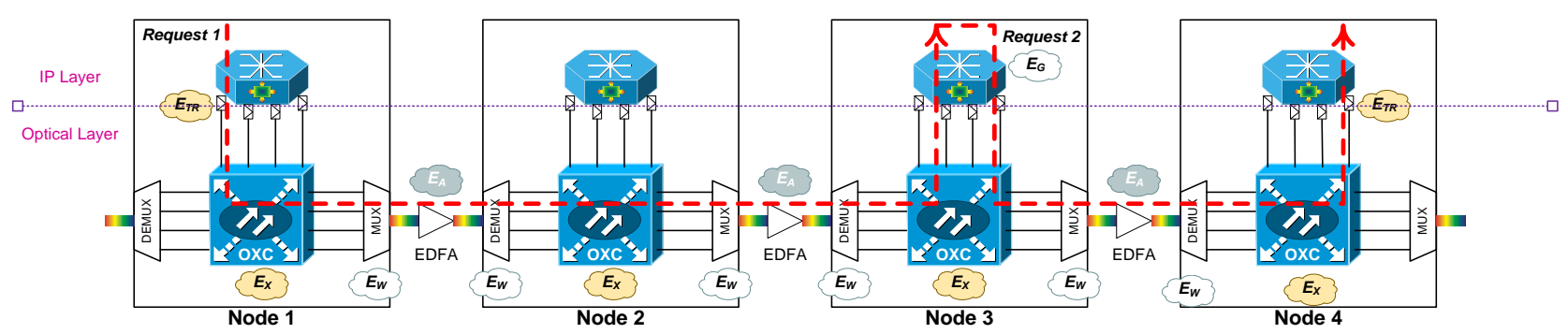

Fig. 1. Transparent IP over WDM architecture.

Transmission of traffic can be accomplished in either the electronic or optical domains; the former is implemented in the core router through traffic grooming and optical signal regeneration; the latter can be achieved by $\mathrm{OXCs}$ providing that no regeneration is required, bypassing light paths at intermediate core routers.

The key contributors to energy consumption are WDM multiplexers/de-multiplexers $\left(\mathrm{E}_{\mathrm{W}}\right)$, optical transponders $\left(\mathrm{E}_{\mathrm{TR}}\right), \operatorname{OXCs}\left(\mathrm{E}_{\mathrm{X}}\right)$, EDFAs $\left(\mathrm{E}_{\mathrm{A}}\right)$ and core routers $\left(\mathrm{E}_{\mathrm{G}}\right)$.

\section{NETWORK ENERGY MODEL}

In our approach in order to evaluate overall network power consumption and consumed energy per a data bit we used so called equivalent network energy model (see Fig. 2) based on multilayer Internet Protocol / Generalized Multi-Protocol Label Switching (IP/GMPLS) over optical layers. In this model, a network carrier bandwidth of OC-192 and average energy consumption of 1019nJ per bit was assumed following [6]-[8].

The parameter $G_{n}$ denotes a Router's dissipated power of $10 \mathrm{~kW}$ within its energy consumption of $1000 \mathrm{~nJ} / \mathrm{bit} . \mathrm{X}_{\mathrm{n}}$ represents Optical Cross-Connect (OXC) dissipating $100 \mathrm{~W}$ and consumes $10 \mathrm{~nJ} / \mathrm{bit}$. $\mathrm{W}_{\mathrm{n}}$ denotes Wavelength Division Multiplexing (WDM) part of the node with dissipating power of $120 \mathrm{~W}$ and energy consumption of 12nJ/bit. $A_{n}$ represents the consumption owing to Erbium-Doped Fibre Amplifiers (EDFA) within 
connection spans placed at $70 \mathrm{~km}$ intervals, the power consumption is estimated to be $1 \mathrm{~W}$ with energy of $0.1 \mathrm{~nJ} / \mathrm{bit}$.

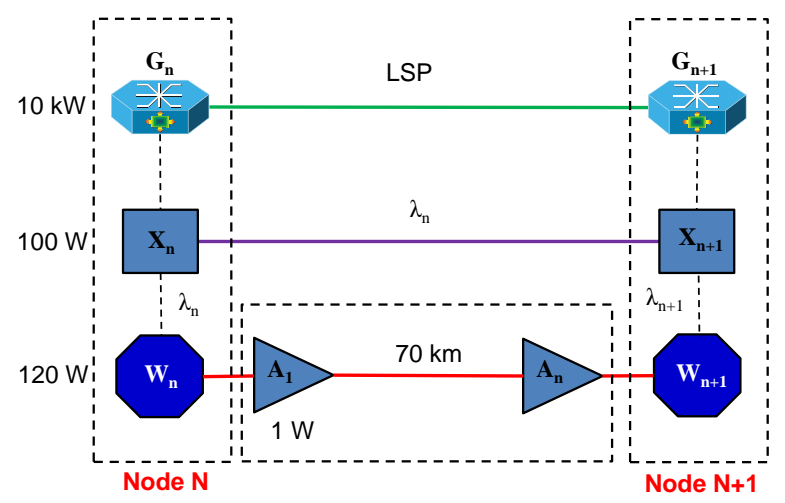

Fig. 2. Energy model for transparent optical IP network.

\section{A. Energy per Bit}

Further we define energy per bit consumed by the node as $\mathrm{E}_{\mathrm{b}}=\mathrm{P}_{\mathrm{T}} / \mathrm{C}$ where $\mathrm{P}_{\mathrm{T}}$ represents the node total power consumption and $\mathrm{C}$ is the bandwidth offered by the network link.

\section{B. Power Consumption}

The total power consumption of the link is given by:

$$
P_{T}=P_{g}+P_{x}+P_{a}+P_{w}+P_{t r}
$$

where $\mathrm{P}_{\mathrm{g}}, \mathrm{P}_{\mathrm{x}}, \mathrm{P}_{\mathrm{a}}, \mathrm{P}_{\mathrm{w}}$, and $\mathrm{P}_{\text {tr }}$ represent power consumed by the IP/GMPLS router, OXC, EDFA, WDM, and transmitter, respectively.

\section{Energy Efficiency}

The energy efficiency in optical IP networks can be measured based on the system capacity [7], [9] as it is shown in Table I bellow.
The throughput is defined as the rate of successful data or message delivery flowing through the network (physical or logical link) and is measured in bits per second (bit/s). Goodput represents the number of actual useful bits per unit of time (omitting the overhead and retransmitted data packets). It's determined as the ratio of the delivered data payload to the transmission time.

\section{Energy Saving Duration}

The Finite State Machine (FSM) is an important component within the energy saving model, playing a key role in determining state transitions from the $\mathrm{ON}$ to the OFF state.

Fig. 4 demonstrates the FSM ON-OFF process model assuming finite variance distributions for the $\mathrm{ON}$ and OFF periods where both ON/OFF are two independent processes. The model treats the ON, Hibernation Mode (HM) and OFF states.

At the beginning of the simulation, all links and wavelengths are free. LSPs are only generated during the $\mathrm{ON}$ state following a fixed inter-arrival time. The ON state represents active traffic activity, returning to Busy on the ON condition. When a request arrives during the ON state, the LSP request moves the node state to HM by triggering Resv_Confirm.

Consider the energy saving duration pattern shown in Fig. 4. The Hibernation inter-activity interval (OFF State) is defined as:

$$
T_{H}=\sum_{\text {path-node }}^{n}\left(\delta T_{s}+\tau_{\text {off }}+\delta T_{w}\right)
$$

and

$$
\left(\delta T_{s}+\delta T_{w}\right)<T_{H}
$$

TAble I: ENergy EfFiciency in Optical IP Networks. $\eta$ IS Energy Efficiency (Joule Per Bit). $P_{T}$ STANDS for Total System Power (IN WATT). C REPRESENTS SYSTEM CAPACITY (IN BITS/SECOND). $\gamma$ IS SYSTEM THROUGHPUT (IN BITS/SECOND). G IS SySTEM GOODPUT (IN BITS/SECOND).

\begin{tabular}{|l|c|}
\hline \multicolumn{1}{|c|}{ Energy Efficiency $(\boldsymbol{\eta})$} & Formulae \\
\hline Measurement based on Capacity & $\eta_{C}=\frac{P_{T}}{C}$ \\
\hline Measurement based on a Throughput (aggregate) & $\eta_{\gamma}=\frac{P_{T}}{\gamma}$ \\
\hline Measurement based on a Goodput (actual useful bits) & $\eta_{G}=\frac{P_{T}}{G}$ \\
\hline
\end{tabular}

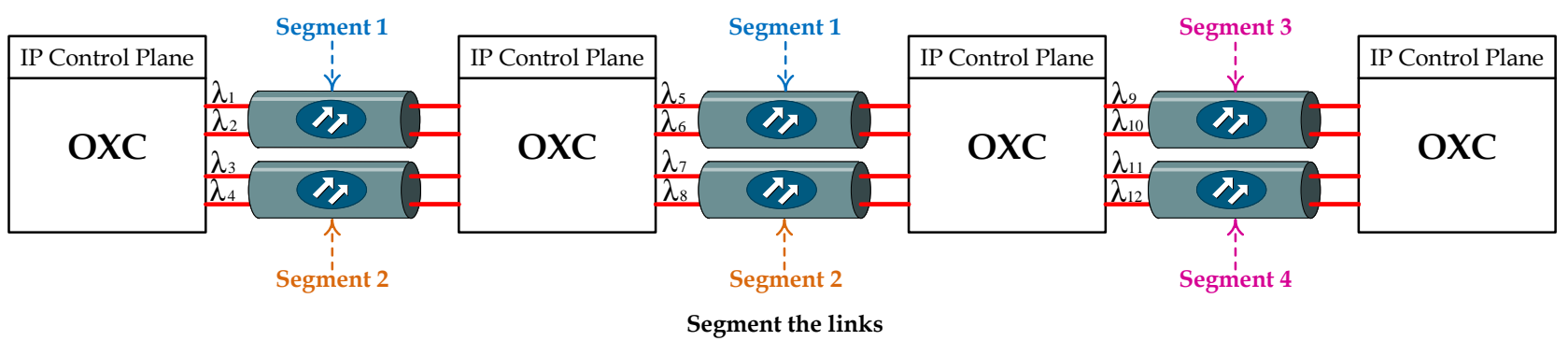

Fig. 3. Segmentation-link hibernation mode structure. 
If the variables $\delta T_{s}$ and $\delta T_{w}$ are independent and specified as $\left(\delta T_{s}+\delta T_{w}\right)<T_{H}$, then it is possible to turn off network equipment and save energy with no increase in delay (or by increasing refresh time for the Path and Resv messages).

However, since in the optical core network provisions high link capacity, and when the variables $\delta T_{s}$ and $\delta T_{w}$ are small i.e. $\left(\delta T_{s}+\delta T_{w}\right) \rightarrow 0$, they are assumed to be negligible.

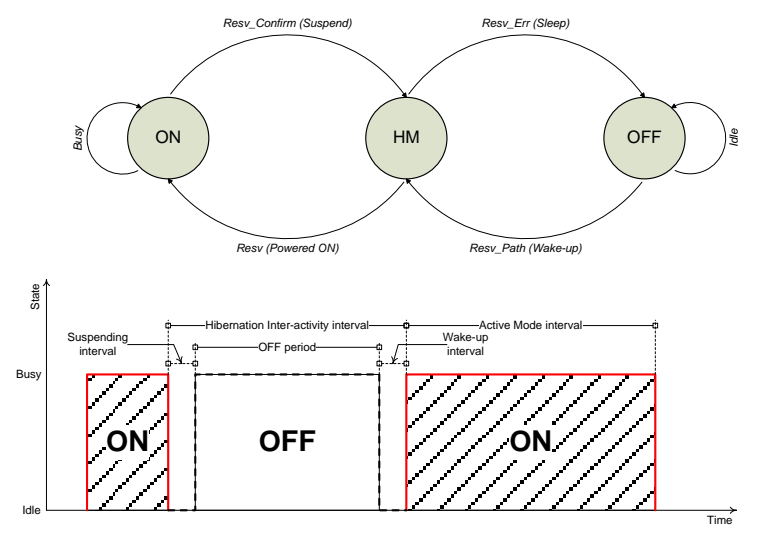

Fig. 4. Energy saving duration process.

\section{HibERnATION MODE WITH SEGMENTATION-LINK MECHANISM}

Fig. 3 presents the Segmentation-Link structure of the hibernation strategy. In this architecture, nodes are interconnected by point-to-point optical fibre links and a control plane link is used to propagate IP-based control messages, including routing and signalling.

A reduction in power consumption is implemented by re-configuring fibre links to segment traffic flows e.g. rerouting all traffic to light paths at segment 1 (active wavelengths) while re-routing the inactive wavelengths to segment 2 (idle wavelengths). Power consumption can be distributed based on fibre link selection and re-routing the traffic via the OSPF-TE extension, already used to advertise the hibernation mode.

\section{A. Power Saving using Optical Bypass}

Optical IP networks have the capability to optically bypass the $\mathrm{OXC}$ through reconfiguration at intermediate nodes, in so doing reducing the level of electronic processing at the core router with a concomitant lowering of the power consumption.

In addition to, the optical bypass in OXC overlay link to core IP router will reduced the required number of IP router ports/interfaces can potentially maximally save the energy consumption of an IP over WDM networks [10].

\section{B. Power Saving with Traffic Grooming}

Energy-aware traffic grooming techniques have potentially reduce significant amount of energy consumption in IP over WDM networks. To enable the traffic grooming mechanism, the lightpaths consisting of connected multiple links are established constructing virtual topologies. A lightpath of traffic grooming is a sequence of unidirectional links which can use different wavelengths. Optical nodes have wavelength conversion capability so that input wavelength channels of a node can be converted into different output wavelength channels [11]. Traffic grooming techniques is employ to optimum the optical transport system utilization.

\section{Simulation RESUlts}

Fig. 5 illustrates the network topology which is being utilised by the European Optical Network (EON network). The EON network has a Full Mesh Network Topology with 9 nodes and 20 bidirectional fibre links [10], [11]. The IP/GMPLS nodes are linked by bidirectional pairs of single mode fibres. The EON network topology was used in our simulations and was based on a discrete event modelling tool known as OMNet++ (Object Modular Network Tested in $\mathrm{C}++$ ). It has been assumed that all links are equal in terms of number of wavelengths (eight), that the message length is fixed at 256 bytes, and a nodal processing delay is $20 \mathrm{~ms}$. All EON network Nodes are capable to maintain information on their total power consumption as well as energy per bit consumed. Wherein, the standard GMPLS signalling and routing protocols are implemented following the Internet Engineering Task Force (IETF) standard [12][13].

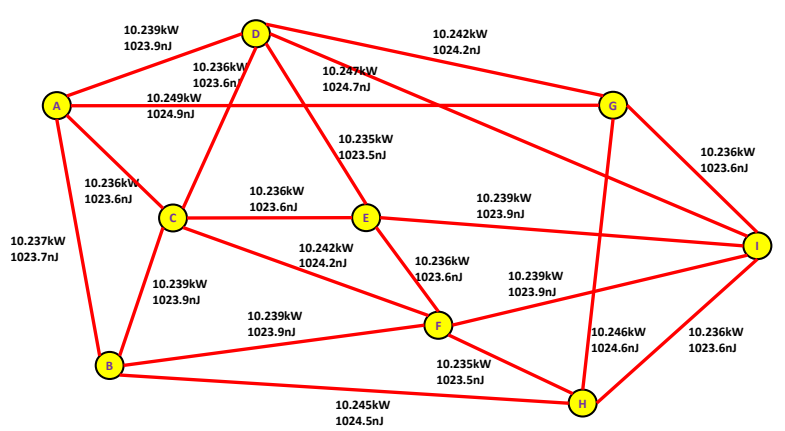

Fig. 5. European optical network (EON) full mesh topology.

The performance metrics takes into account the average power consumption, blocking probability and average request blocking [9], [12]-[15]. We also assumed that lightpath requests are uniformly distributed. Note that, the inter-arrival connection requests are independent Poisson processes with an arrival rate of $\alpha$ and the queue lengths exponentially distributed with the expected service rate time of $1 / \mu$ measured in seconds. Therefore, the network offered load is $\alpha / \mu$.

The proposed Hibernation concept was verified on described EON by implementing segmentation-link schemes. These nodes/links are then put into "hibernation" or an "SLEEP state", in which nodes have suspended their unused functionalities (e.g. unused ports / interfaces, Mux/DeMux capabilities, signalling gates, unused wavelengths, etc.) and keep only the minimum network operation activities.

Fig. 6 presents the average power consumption for various segment-link settings as a function of offered network load. The average power difference is defined as 
the difference between the maximum power consumption (reference power) by a node to the power dissipation of the node under the energy saving scheme. When the network utilises the hibernation mode, the power saving for a node is $0.176 \mathrm{~kW}$ (link-only scheme) and $0.221 \mathrm{~kW}$ (segment link-scheme) at 2 Erlangs at a corresponding blocking probability of $24 \%$ and $40 \%$ respectively. The OXC makes use of the all-optical wavelength converters to reduce power dissipation through $\mathrm{HM}$ and ON states; the former de-activates wavelengths and the latter activates wavelengths. The segment-link scheme bundles individual links, in so doing aggregating the available resources and improving routing scalability by reducing the amount of information to be processed by the control plane.

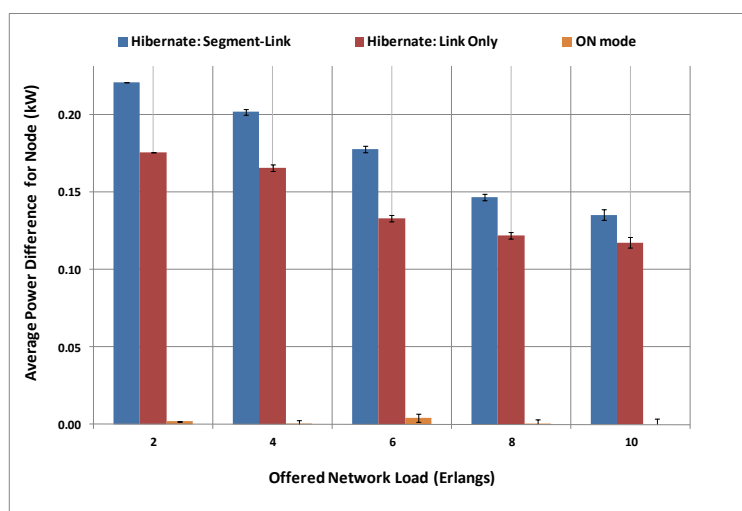

Fig. 6. Energy average power difference as a function of different segment-link schemes of operation.

Therefore, the number of routing adjacencies in the network is proportional to the number of control planes connected to the nodes and is not governed by the number of data plane links. Consequently, network resources are optimized, reducing the power consumed.

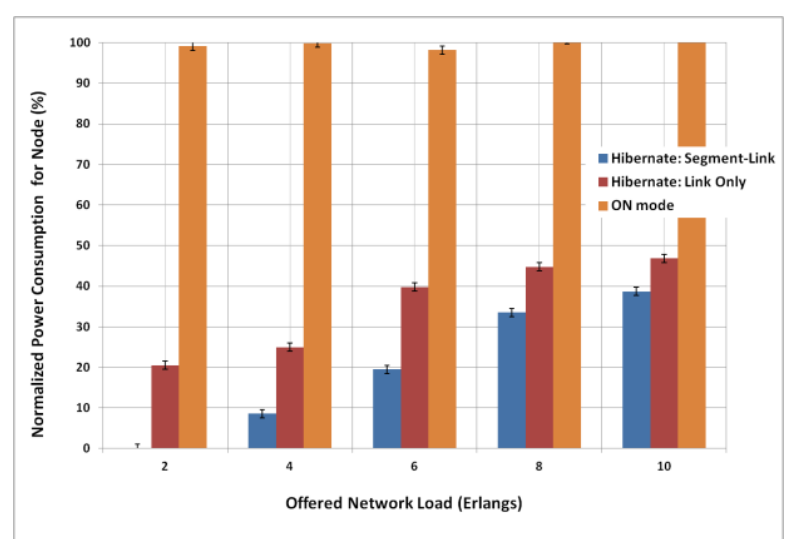

Fig. 7. Normalized power consumption for various segment-link settings as a function of offered network load.

Fig. 7 shows the normalised power consumption of different hibernation segment-link schemes normalised to the reference "ON" state as a function of offered network load. Results indicate that a segment-link scheme can yield a power saving of $67 \%$ at 8 Erlangs compared to the link only scheme (55\%). Fig. 8 presents the percentage of power consumed by the Optical and IP domain network equipment respectively when the segment-link scheme is applied. The Power Ratio is the ratio of the number connections under segment-link modes over the number of the active ON state nodes. The results show that both the segment-link scheme and the 'link only' scheme in optical domain can yield a power savings of $60 \%$ and $50 \%$ respectively at a network load of 8 Erlangs.

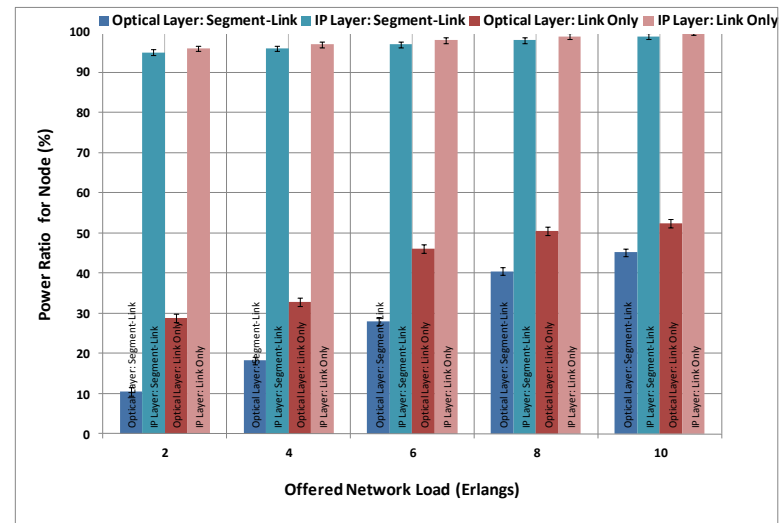

Fig. 8. Comparison of the power ratio between optical layer and IP layer for segment-link schemes as a function of offered network load traffic.

The impact on blocking probability of the link-only and segment-link schemes is show in Figure 9. In the 'link only scheme' the probability of blocking is below $24 \%$ (Fig. 9) thus delivering a better network performance but the power reduction/savings is minimal at $0.176 \mathrm{~kW}$. The segment-link scheme yields a blocking probability of $\sim 40 \%$ and offers $0.221 \mathrm{~kW}$ of power saving The OXC makes use of all-optical wavelength converters to reduce blocking probability.

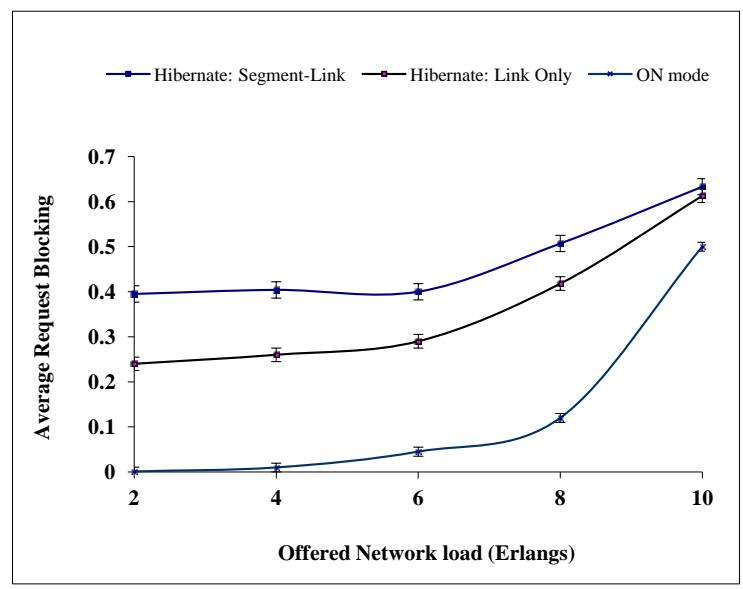

Fig. 9. Request blocking for different Segment-Links schemes.

Fig. 10 presents the link power consumption as a function of the degree of connectivity $\mathrm{D}$. The results shows that the when 8 wavelengths are available and the traffic is at the peak level, D equals to 2 leading to a power saving (normalized) of about $60 \%$ and $80 \%$ for the link only and segment-link schemes, respectively; values of D greater than 2 do not result in any decrease in the power consumed. It is clear that high values of $\mathrm{D}$ lead to a better network capacity utilisation and concomitant lower power consumption. 


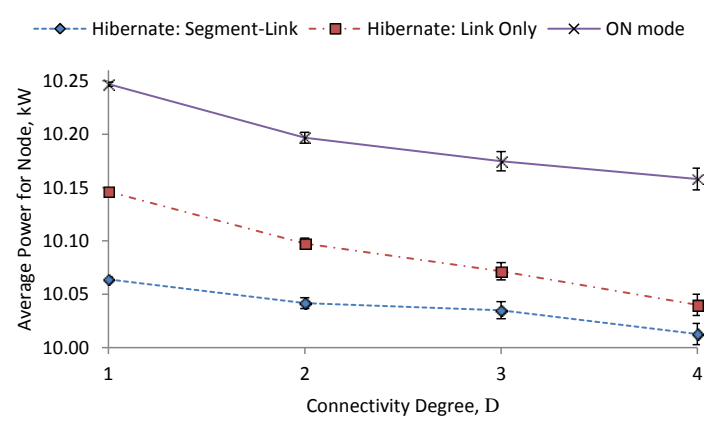

Fig. 10. Link power consumption as a function of the degree of network connectivity for peak and minimum traffic loads for different SegmentLinks schemes.

Fig. 11 provides the power ratio difference for a node for both the optical and IP layers as a function of the degree of network connectivity when segmentation-link schemes are applied. The power ratio here is defined as the power consumption ratio for nodes of selected light paths to the degree of network connectivity. The results show that in optical domain the segment-link scheme yields a higher power saving ratio $(\sim 75 \%)$ than the link only scheme $(\sim 40 \%)$. The reason for this is that the segment-link scheme utilises power-aware-routing based on OSPF-TE standards to re-route light paths as the network connectivity degree escalates. In addition, the results demonstrate that the power savings ratio in the IP domain is slightly increased (5\%) as compared to link only scheme (2\%); the core routers with segment-link schemes apply traffic grooming and adaptive OSPF-TE routing.

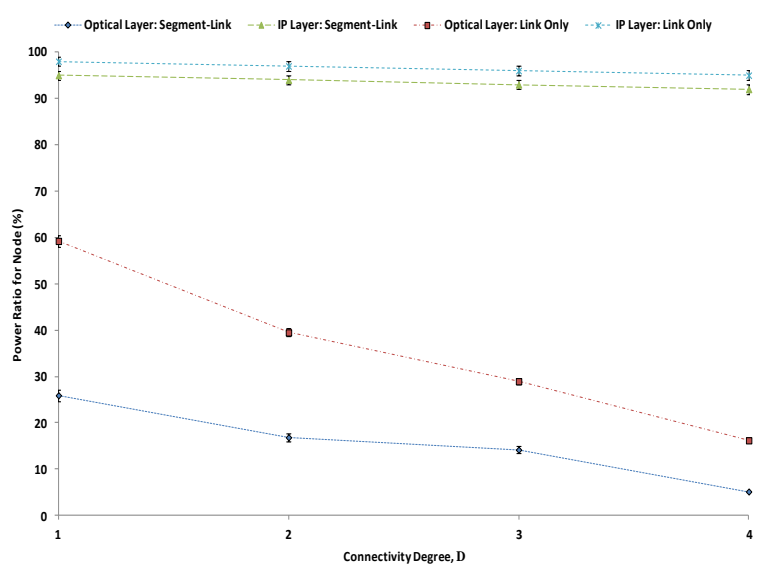

Fig. 11. Link power consumption as a function of the degree of network connectivity for peak and minimum traffic loads for different SegmentLinks schemes.

Fig. 12 presents the power consumption as a function of the number of nodes for different segment-links schemes. The results demonstrate that the segment-link scheme yields a higher power saving than link only scheme; the latter utilises power-aware-routing based on OSPF-TE standards to re-route the light paths as the network size grows.

Fig. 13 presents the power ratio for a node for both the optical and IP layers as a function of the number of nodes.
The power ratio is defined as the ratio of the power consumption of nodes of selected fibre links to the number of adjacent nodes. The results show that the power ratio at optical layer improves from small to large network sizes when the segment-link scheme is applied and yields a higher power saving ratio (from $~ 60 \%$ up to $95 \%$ ) than the link only scheme (from $\sim 48 \%$ up to $95 \%$ ). Notice that when the number of nodes is $k=1$ (small network size) the power ratio for both schemes is similar but decreases as the network size grows. Moreover, the results demonstrate that the power saving ratio at the IP layer on the segment-link slightly increases (5\%) whilst for the link only scheme declines $(2 \%)$.

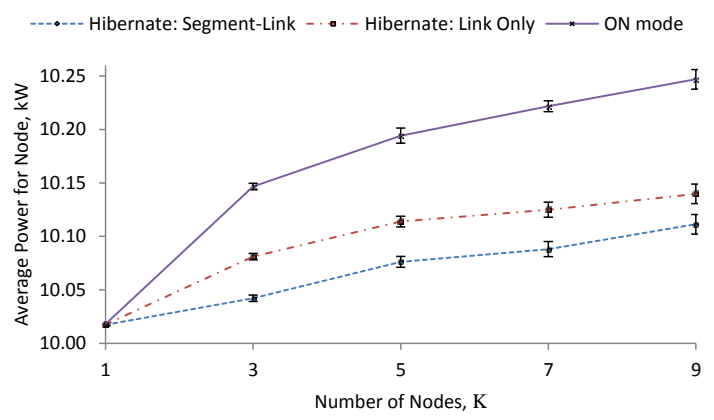

Fig. 12. Power consumption as a function of the number of nodes for different segment-links schemes.

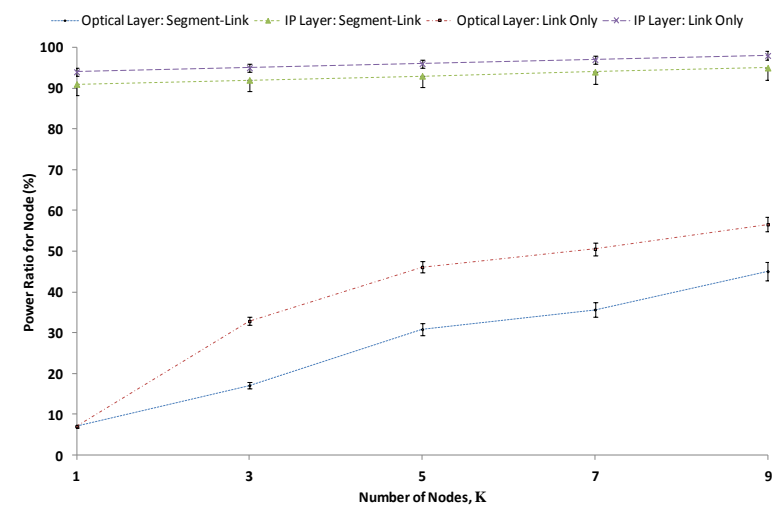

Fig. 13. Power ratio versus number of nodes for different schemes of segment-links operation.

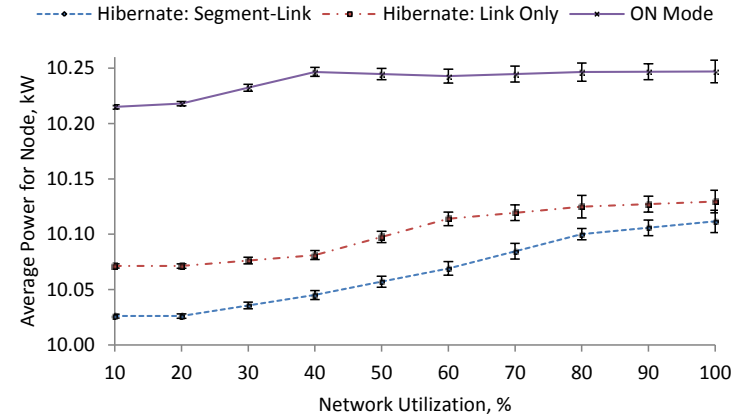

Fig. 14. Power consumption as a function of network utilisation for different Segment-Links schemes.

Fig. 14 demonstrates the power consumption as a function of network utilization. Results show that the 
segment-link scheme yields a significant saving in power consumed as network utilization (network offered load) increases; the average power consumption grows with network utilization and network size. The segment-link scheme yields a more marked improvement on average power consumption in proportion to network utilization compared to link only scheme - with $\sim 10.025 \mathrm{~kW}$ power dissipated - owing to minimal traffic disruption when fibre links are powered down and LSP requests optimized.

Fig. 15 presents the power ratio for a node for both the optical and IP layers as a function of network utilization. The power ratio is defined as the ratio of the power consumption of nodes of selected hibernation fibre links over the network utilization. The results show that the power ratio at the optical domain improves when the segment-link scheme is applied and yields a higher power saving ratio (from $40 \%$ up to $\sim 90 \%$ ) than the link only scheme (from $~ 50 \%$ up to $71 \%$ ). Additionally, the results demonstrate that the power ratio at the IP domain with segment-link steadily rises $(10 \%)$ in contrast to link only scheme $(4 \%)$. The core routers with the segment-link scheme apply traffic grooming for low-rate traffic; for high-rate traffic, segmentation/link aggregation and adaptive OSPF-TE routing are applied at the IP domain.

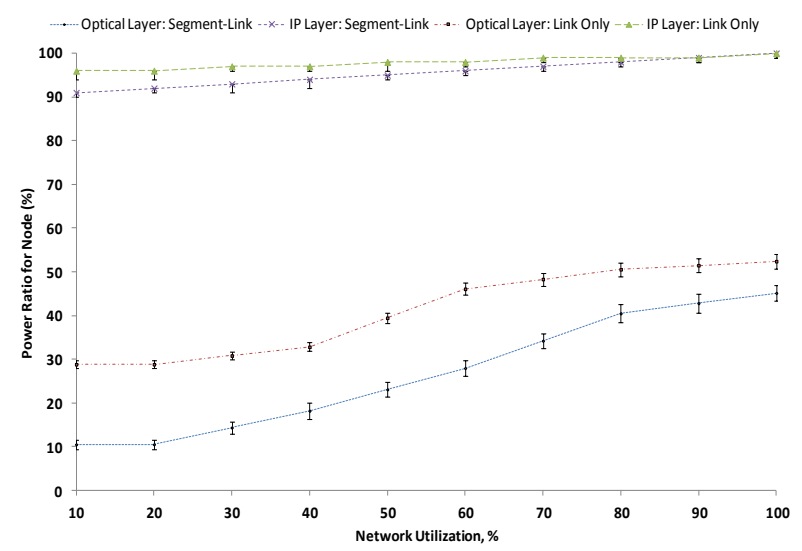

Fig. 15. Power ratio as a function of network utilisation for different Segment-Links schemes.

\section{CONCLUSIONS}

In this paper we have presented a new energy conservation concept which delivers improved network power management. Issues that were addressed in this study were segmentation-link schemes. The proposed "Hibernation mode" technique can lower energy consumption in multilayer optical IP networks. Segmentlink is a technique that identifies and powers down idle optical fibre links for the purposes of energy conservation. The mechanism aims to minimise power consumption in both the optical and IP domains; the former utilises optical bypass at intermediate OXC with light pathrerouting while in the latter traffic grooming and low-rate traffic aggregation is used.

With segment-link, the energy saving is based on the generic "Hibernation Mode" in tandem with "Routing and Wavelength Assignment". The former can be further broken down as segment-links and link only schemes. The latter harnesses heuristic algorithms to underpin power consumption minimization taking into account segment-link and wavelength continuity constraints. To demonstrate its effectiveness, the method was applied to selected fibre links/nodes and then the average power consumption versus network performance was simulated on European Optical Network topology. In summary, we have investigated the impact of the energy proportionally of network links against number of connectivity degree, probability of blocking, normalized power consumption and power ratio. Obtained results showed that a significant amount of energy can be saved if an appropriate "segmentation-link" configuration is invoked.

\section{ACKNOWLEDGMENT}

The project in this study has been designed using OMNET++ simulator. It was supported in part by a grant from the Ministry of Higher Education Malaysia and the University Malaysia Perlis under the RAGS grant number of 9018-0003.

\section{REFERENCES}

[1] CISCO Visual Networking Index - Forecast and Methodology 2010-2015. (Jun 2011). [Online]. Available: http://www.cisco.com

[2] K. Manousakis, A. Angeletou, and E. M. Varvarigos, "Energy efficient RWA strategies for WDM optical networks," IEEE J. Opt Communication Networks, vol. 5, no. 4, pp. 338-348, April 2013

[3] K. Pawlikowski, H. D. Joshua Jeong, and J. S. Ruth Lee, "On credibility of simulation studies of telecommunication networks," IEEE Communications Magazine, vol. 40, no. 1, pp. 132-139, Jan 2002.

[4] F. Musumecci, M. Tornatore, and A. Pattavina, "A power consumption analysis for IP-Over-WDM core network architecture," IEEE/OSA Journal of Optical Communications and Networking, vol. 4, no. 2, pp. 108-117, Feb 2012.

[5] B. Rajagopalan, J. V. Luciani, and D. O. Awduche, "IP over optical networks: A framework," RFC 3717, Internet Engineering Task Force, March 2004.

[6] J. Balinga, R. Ayre, K. Hinton, W. V. Sorin, and R. S. Tucker, "Energy consumption in optical IP networks," IEEE J. Lightwave. Technol. vol. 27, no. 13, pp. 2391-2403, 2009.

[7] B. G. Bathula, M. Alresheedi, and J. M. H. Elmirghani, "Energy efficient architectures for optical networks," in Proc. London Communications Symposium (University College London), London, 2009, pp. 1-4.

[8] Cisco Systems Data Sheets. (Sept 2012). [Online]. Available: http://www.cisco.com

[9] S. J. Ben Yoo. "Energy Efficiency in the future internet: The role of optical packet switching and optical-label switching," IEEE Journal of Selected Topics in Quantum Electronics, vol. 17, no. 2, pp. 406-418, Mar/April 2011.

[10] G. Shen and R. S. Tucker, "Energy-minimized design for IP over WDM networks," IEEE/OSA Journal of Optical Communications and Networking, vol. 1, no. 1, pp. 176-186, June 2009.

[11] Y. Kim, C. Lee, J. K. Kevin Rhee, and S. Lee, "IP over WDM cross-layer design for green optical networking with energy proportionally consideration," IEEE Journal of Lightwave Technology, vol. 30, no. 13, pp. 2088-2096, July 2012. 
[12] S. Albarrak, "Failure recovery in distributed GMPLS-based ipover-optical networks," Ph.D. Thesis, Dept. Electronic \& Elect. Eng., Univ. of Strathclyde, Glasgow, UK, 2008.

[13] J. Chabarek, J. Sommers, P. Barford, C. Estan, D. Tsiang, and S. Wright, "Power awareness in network design and routing," in Proc. 27th Conf. on Computer Communications, Phoenix, 2008, pp. 1130-1138

[14] L. Berger, "Generalized multi-protocol label switching (GMPLS) signalling functional description," RFC 3471, Internet Engineering Task Force, 2003.

[15] J. Lang, "Generalized multi-protocol label switching (GMPLS) signalling resource reservation protocol-traffic engineering (RSVP-TE) extension," RFC 3473, Internet Engineering Task Force, 2003.

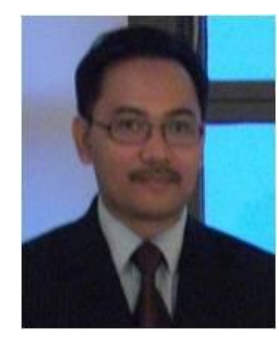

Mohd Nazri Bin Mohd Warip received BEng (Hons) in Electronic and Electrical Engineering from University of Portsmouth, United Kingdom. MEng in Electrical, Electronics \& Telecommunications from University of Technology Malaysia and $\mathrm{PhD}$ from University of Strathclyde, Glasgow. Heis a Senior Lecturer at School of Computer and Communication Engineering, Universiti Malaysia Perlis. His current research interests are focusing on IP over WDM, GMPLS, 100 gigabit Ethernet transport, passive optical networks, broadband networks, grid networks, network architecture and cross-layer interaction design, optical network modeling, green networks, energy harvesting and emerging trends in future core/metro/access networks technologies.

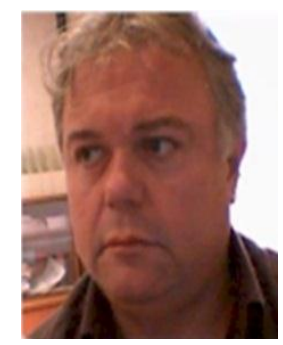

Ivan Andonovic BSc, $\mathrm{PhD}$, CEng, FIET, SMIEEE, MOSA. He is a professor and head of CIDCOM (Centre for Intelligent Dynamic Communications), University of Strathclyde, Glasgow, United Kingdom. He is also a BT Short Term Fellow; Royal Society/ EPSRC Industrial Fellow; IET Faraday Lecturer; Visiting Scientist Communications Research Labs. of Japan; Topical Editor IEEE Transactions on Communications; Director of Embedded Technology Solutions (ETS); Visiting Professor, Princeton University; Technical Co-Chair IEEE ICC2007 His research interests are optical networks, photonic switching and routing, optical code division multiple access, wireless sensor networks, wireless ad hoc networking.

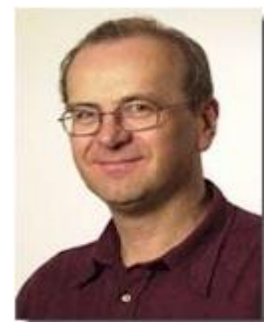

Ivan Glesk D.Sc, $\mathrm{PhD}$. He is a Professor and Director of CIDCOM Laboratories. He is also a Head of the Joint GRPe Graduate School, University of Strathclyde, Glasgow, United Kingdom. He joined the Department of Electronic and Electrical Engineering University of Strathclyde in 2007. Between 1991 and 2007 he was Senior Research Scholar and the Manager of the Lightwave Communication Research Laboratory in the
Department of Electrical Engineering at Princeton University, USA. As the recipient of IREX Fellowship he was a Visiting Fellow at the Department of Mechanical and Aerospace Engineering at Princeton University in 1990. He also held a position Professor of Physics at Comenius University in Bratislava. His research interests are Broadband communication systems, data and transmission security, optical interconnects, optical amplifiers, short pulse fiber lasers, WDM, OTDM, OCDM, optical switching and routing, optical and all-optical devices, nanowire/subwavelength-based devices and sensors, LIDAR sensing, and bio photonics.

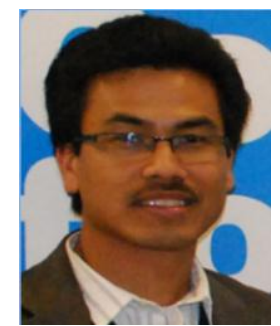

Phaklen Ehkan received the B.Eng degree in electrical engineering from University of Technology Malaysia, M.Sc in information technology from Northern Malaysia University and Ph.D degree in computer engineering from the University Malaysia Perlis - University of Birmingham, United Kingdom. He joined School of Computer and Communication Engineering, University Malaysia Perlis in 2003. He engaged in research on the embedded system, digital system design using FPGA, DSP, speech analysis and recognition. Phaklen is a member of IEEE, BCS and IACSIT.

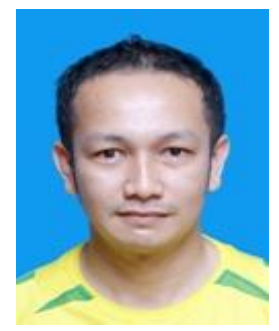

Fairul Afzal Ahmad Fuad received the B.Eng degree in communication engineering from University of Toukai, Japan and M.Sc in electronics system design engineering from University Science Malaysia. He is a Lecturer under School of Computer and Communication Engineering, University system design. Malaysia Perlis. Currently, he is working on embedded and electronic

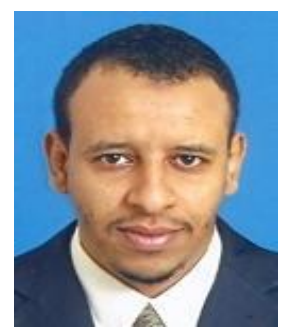

Mohamed Elshaikh Elobaid Said Ahmed is a Lecturer under School OF Computer and Communication Engineering, University Malaysia Perlis, Malaysia. He received M.Sc Electrical and Electronics Engineering from University Technology Petronas (UTP), Malaysia and B.Sc Engineering Technology (Computer Engineering), University of Gezira, Sudan. His research mainly is on computer networking related. 